\title{
Propuesta de calificación en turismo en el territorio brasileño de la Ruta Bioceánica a la luz de la Política Nacional de Calificación en Turismo (PNCT)
}

\author{
Proposta de qualificação em turismo no território brasileiro da Rota Bioceânica \\ à luz da Política Nacional de Qualificação em Turismo (PNQT)
}

\author{
Proposal for qualification in tourism in the Brazilian territory of the Bioceanic \\ Route in light of the National Qualification Policy in Tourism (NQPT)
}

\author{
Dyego de Oliveira Arruda ${ }^{1}$ \\ Patricia Zaczuk Bassinello \\ Carla Cristina de Souza ${ }^{3}$ \\ Milton Augusto Pasquotto Mariani ${ }^{3}$
}

Recibido el 14 de mayo de 2021; revisado e aprobado el 7 de sept. de 2021; aceptado el 13 de oct. de 2021 DOI: http://dx.doi.org/10.20435/inter.v22i4.3376

\begin{abstract}
Resumen: Este artículo tiene como objetivo presentar una propuesta para la difusión de acciones de capacitación profesional, técnica y humanística dirigidas al turismo en el territorio brasileño comprendido por la Ruta Bioceánica, teniendo como trasfondo las premisas fundamentales expresadas en la Política Nacional de Calificación en Turismo (PNCT). En general, vale decir que PNCT determinó un conjunto de directrices para las estrategias de calificación turística en Brasil, con el fin de incrementar la competitividad e importancia socioeconómica de esta actividad en el país. En términos metodológicos, se llevó a cabo una investigación con un enfoque cualitativo, en la que se realizaron seis ruedas de conversación con diferentes actores relacionados con el turismo en el territorio brasileño de la Ruta Bioceánica. Las ruedas de conversación, realizadas virtualmente, fueron grabadas, transcritas y analizadas a la luz del discurso expresado por los actores del turismo que participaron en los diálogos e interacciones promovidas a lo largo de la investigación. En resumen, los principales resultados del estudio apuntaron la existencia de tres ejes temáticos (Ecosistema Turístico, Cultura y Gobernanza y Tecnología) que estructuran las posibles acciones de habilitación de la Ruta Bioceánica. Además, el artículo también reveló la importancia de los órganos de gobernanza local/regional (como la Red Universitaria de la Ruta de Integración Latinoamericana- UniRila), además de la necesidad de insertar a las comunidades tradicionales en los procesos de calificación en turismo en el territorio brasileño de la Ruta Bioceánica.
\end{abstract}

Palabras clave: Ruta Bioceánica; calificación en turismo; políticas públicas; desarrollo local.

Resumo: O presente artigo tem como objetivo apresentar uma proposta para a difusão de ações de qualificação profissional, técnica e humanística voltadas para o turismo no território brasileiro compreendido pela Rota Bioceânica, tendo como pano de fundo as premissas fundamentais expressas na Política Nacional de Qualificação em Turismo (PNQT). De uma maneira geral, vale dizer que a PNQT determinou um conjunto de direcionamentos para as estratégias de qualificação em turismo no Brasil, de modo a incrementar a competitividade e a importância socioeconômica desta atividade no país. Em termos metodológicos conduziuse uma pesquisa de abordagem qualitativa, em que foram realizadas seis rodas de conversa com distintos atores ligados ao turismo no território brasileiro da Rota Bioceânica. As rodas de conversa, realizadas de forma virtual, foram gravadas, transcritas e analisadas à luz das discursividades manifestadas pelos atores do turismo que participaram dos diálogos e interações promovidos ao longo da pesquisa. Em síntese, os principais resultados do estudo apontaram para a existência de três eixos temáticos (Ecossistema Turístico, Governança e Tecnologia e Cultura) estruturantes das possíveis ações de qualificação para a Rota Bioceânica. Além disso, o artigo também revelou a importância das instâncias de governança locais/regionais (tais como a Rede Universitária da Rota de Integração Latino-Americana - UniRila), além da necessidade de inserção das comunidades tradicionais nos processos de qualificação em turismo no território brasileiro da Rota Bioceânica. Palavras-chave: Rota Bioceânica; qualificação em turismo; políticas públicas; desenvolvimento local.

\footnotetext{
${ }^{1}$ Centro Federal de Educação Tecnológica Celso Suckow da Fonseca (CEFET/RJ), Valença, Rio de Janeiro, Brasil.

${ }^{2}$ Universidade Federal de Mato Grosso do Sul, Campo Grande, Mato Grosso do Sul, Brasil.

${ }^{3}$ Universidade Católica Dom Bosco, Campo Grande, Mato Grosso do Sul, Brasil.
} 


\begin{abstract}
This article aims to present a proposal for the dissemination of professional, technical and humanistic qualification actions focused on tourism in the Brazilian territory comprised by the Bioceanic Route, with the backdrop of the fundamental premises expressed in the National Qualification Policy in Tourism (NQPT). In general, it is worth saying that the NQPT determined a set of guidelines for qualification strategies in tourism in Brazil, in order to increase the competitiveness and socioeconomic importance of this activity in the country. In methodological terms, a qualitative research was conducted, in which six conversations wheels were carried out with different actors linked to tourism in the Brazilian territory of the Bioceanic Route. The conversation wheels, carried out remotely, were recorded, transcribed and analyzed in the light of the discursivities manifested by the tourism actors who participated in the dialogues and interactions promoted throughout the research. In summary, the main results of the study pointed to the existence of three thematic axes (Tourist Ecosystem, Culture and Governance and Technology) structuring the possible qualification actions for the Bioceanic Route. Furthermore, the article also revealed the importance of local/ regional governance instances (such as the University Network of the Latin American Integration RouteUniRila), as well as the need for insertion of traditional communities in tourism qualification processes in Brazilian territory of the Bioceanic Route.
\end{abstract}

Keywords: Bioceanic Route; qualification in tourism; public policies; local development.

\title{
1 INTRODUCCIÓN
}

La Política Nacional de Calificación Turística (PNCT) es un documento rector, elaborado bajo la coordinación del Ministerio de Turismo de Brasil, cuyo propósito es impulsar el desarrollo de acciones de calificación profesional, técnica y humanística en varios estados y municipios brasileños, con la perspectiva de la creación de oportunidades de empleo y la mejora de los productos y servicios ofrecidos en los destinos turísticos (BRASIL, 2018a).

Se sabe que el turismo, como actividad socioeconómica, es una importante fuente de empleo y generación de ingresos. En 2019, antes de la aparición de la pandemia de Covid-19, que afectó gravemente la actividad turística a escala mundial, el sector de viajes y turismo fue responsable del $10,3 \%$ de toda la riqueza y los ingresos generados en todo el mundo, por lo que la cantidad de puestos de trabajo en actividad directa o indirectamente vinculados al sector turístico en el mundo, superaban a los 330 millones (WORLD TRAVEL \& TOURISM COUNCIL [WTTC], 2021).

En Brasil, en particular, fue de 7,7\% la participación del segmento de viajes y turismo en el total de riqueza e ingresos generados a nivel nacional en 2019, mientras que, en el mismo año, el 7,9\% de todos los empleos formales en el país tuvieron lugar en actividades relacionadas con el turismo (WTTC, 2021). Así, se percibe la magnitud e importancia del turismo en el mundo y en Brasil, en una dinámica en la que las acciones de calificación son cruciales para que esta actividad socioeconómica se consolide de una manera aún más efectiva e impactante, generando oportunidades de empleo e ingresos, especialmente a las personas más vulnerables.

Tomando como trasfondo el tema de la calificación, Silva (2019) considera que PNCT tiene como perspectivas fundamentales: (a) estimular procesos formativos que impliquen la garantía de la ciudadanía y, consecuentemente, en la mejora de la calidad de vida y los niveles de escolarización de las personas; (b) impartir capacitación en turismo de manera integrada con otras modalidades y niveles de enseñanza, incluso en el ámbito de la Educación Básica, respetando las Directrices y Bases de la Educación Nacional y; (c) proponer órganos de gobernanza local y territorial (congregando el gobierno federal, estados y municipios brasileños), a fin de permitir que se lleven a cabo acciones de calificación basadas en las características del turismo realizado sobre bases territoriales.

Desde el punto de vista de la implementación de las acciones de calificación, es importante destacar que PNCT prevé la creación de planes, programas y propuestas regionales de calificación, 
que respeten las características socioculturales, históricas y económicas de los Estados, municipios y otros territorios brasileños, con el fin de estimular con que los técnicos, gestores públicos, empresarios del segmento de turismo, la sociedad civil y las comunidades tradicionales sean involucradas en la planificación y puesta en uso las prácticas turísticas que sean promisoras y sostenibles, en cada una de las localidades brasileñas (BRASIL, 2018).

En ese sentido, ¿Es posible proyectar una propuesta de calificación en turismo que, con base en las premisas de PNCT, permita al territorio brasileño ser un artífice de desarrollo del turismo en la Ruta Bioceánica?

En cierto modo, la Ruta Bioceánica (o Corredor Biooceánico) es una iniciativa sudamericana que tiene como objetivo conectar - especialmente desde el punto de vista de la infraestructura logística - el Atlántico con el Pacífico, congregando territorios de Brasil, Argentina, Paraguay y Chile. Más específicamente, la Ruta Bioceánica forma parte de Campo Grande (capital del Estado de Mato Grosso do Sul, en el Centro Oeste brasileño) y, con una extensión de más de 2.000 kilómetros, tiene como destino la ciudad de Antofagasta, al norte de Chile. Debido al potencial, especialmente vinculado a los activos culturales y socio ambientales de los territorios "conectados" por la Ruta Bioceánica, se sabe que el turismo es una actividad promisora en este contexto, que puede catalizar todo el conjunto de propósitos esperados con la difusión de la Ruta Bioceánica (ASATO et al., 2019).

Castro (2019), recordando el potencial que representa la actividad turística para la Ruta Bioceánica, destaca la relevancia de las acciones de calificación que permiten la inclusión sostenible de personas en el universo de oportunidades generadas por el turismo. El mismo autor también destaca la importancia de la sociedad civil y de las universidades locales, que, sinérgicamente, pueden implicarse en el desarrollo de proyectos de investigación, extensión y formación de recursos humanos para el turismo en los más diversos territorios que abarcan la Ruta.

Por lo tanto, considerando las problematizaciones antes mencionadas, este artículo tiene como objetivo presentar una propuesta para la difusión de acciones de calificación dirigidas al turismo en el territorio brasileño compuesto por la Ruta Bioceánica, en el contexto de las premisas fundamentales expresadas en PNCT.

Es importante destacar que, en este artículo, cuando mencionamos una propuesta de calificación para el turismo en la Ruta Bioceánica, la idea es reunir un conjunto de acciones que, a partir de una investigación empírica (que se detallará más adelante) y las premisas de PNCT, permita el surgimiento de potenciales arreglos institucionales que aumenten la formación y calificación de los recursos humanos para el turismo en la región bajo análisis en este trabajo.

A pesar de que Brasil tiene PNCT debidamente institucionalizada dentro del Ministerio de Turismo, se percibe que el país aún no tiene planes, programas y proyectos (institucionalizados o no) para el desarrollo de acciones específicas de calificación para el turismo emprendido en los más diversos lugares brasileños. En este sentido, entendemos que el esfuerzo analítico de este artículo puede ser importante para que PNCT sea efectivamente implementada y, por lo tanto, para desplegarse en las estrategias locales de capacitación para el turismo en el territorio brasileño abarcado por la Ruta Bioceánica. 


\section{LA CALIFICACIÓN EN TURISMO Y PNCT}

El tema de la calificación es fundamental para incrementar los beneficios potenciales del turismo como actividad que genera empleo e ingresos, en una dinámica en la que se necesitan con urgencia los esfuerzos de los poderes públicos, la iniciativa privada y la sociedad civil para sinergísticamente, planificar y oportunizar actividades de formación en turismo, aumentando los niveles de competitividad de los destinos turísticos.

Catramby y Costa (2004), al examinar las acciones de formación profesional en turismo en Brasil, recuerdan que las actividades de capacitación siempre han ocurrido dentro del Ministerio de Educación (MEC) y el ahora desaparecido Ministerio del Trabajo y Empleo (MTE), sin una política nacional que guiara y coordinara los procesos formativos en el área específica del turismo, una brecha que solo comenzó a ser "equiparada" en los idus de 2018, con la institucionalización de la Política Nacional de Calificación Turística (PNCT) dentro del Ministerio de Turismo.

También según Catramby y Costa (2004, s.p.), históricamente, los procesos formativos en turismo en Brasil siempre han ocurrido através de:

- Cursos superiores, incluyendo cursos de graduación y posgrado (lato sensu o stricto sensu) ofrecidos por instituciones educativas públicas y privadas, respetando las especificidades de la Ley de Directrices y Bases de la Educación Nacional;

- Cursos técnicos, ofrecidos en el ámbito de la Red Federal de Educación Profesional y Tecnológica, así como a través del denominado "Sistema S", que agrupa a instituciones como el Servicio Nacional de Aprendizaje Comercial (SENAC), cuyo enfoque es la formación técnica y profesional para el propio universo del trabajo;

- Cursos libres y no regulares, ofrecidos por instituciones educativas públicas y privadas, así como por la sociedad civil que, a través de Asociaciones y/u Organizaciones No Gubernamentales (ONG), ofrece alternativas de capacitación para grupos específicos, especialmente aquellos que no tienen oportunidades de acceso a cursos superiores y técnicos en turismo.

A partir de 2018, con la institucionalización de PNCT, el tema de la calificación comenzó a ser tomado como estratégico para el conjunto de políticas públicas para estimular el turismo en Brasil. En resumen, PNCT se basa en las siguientes premisas fundamentales (BRASIL, 2018a): (a) la calificación en turismo es una construcción social que debe respetar las especificidades históricas, ambientales y socioculturales de los destinos turísticos; (b) las metodologías de las acciones de calificación deben valorar los conocimientos técnicos de los sujetos y las comunidades, incluidos estos agentes en el ámbito de los beneficios advenidos del turismo; (c) las acciones de cualificación deben entenderse como un camino hacia el desarrollo local/regional y; (d) el locus de las prácticas de capacitación son los territorios, por lo que es importante que PNCT se desarrolle en programas y proyectos específicos que respeten la realidad local y, por lo tanto, promuevan el turismo que es deseable (y deseado) en el territorio.

Vale la pena señalar que PNCT surgió como una rama del Plan Nacional de Turismo (20182022) cuyo lema fundamental es "más empleo e ingresos para Brasil". En este sentido, se percibe que el tema de calificación se revela como estratégico para el logro de las metas expresadas en este documento, ya que las acciones de capacitación son esenciales para la generación de oportunidades de empleo e ingresos en el segmento turístico (BRASIL, 2018b).

La perspectiva local y territorializada para la proposición de acciones de calificación, tal como se expresa en PNCT, es fundamental para que los procesos formativos sean más precisos y, 
por lo tanto, generen menores tasas de evasión y fracaso. En este sentido, Silva y Fratucci (2019) recuerdan que, históricamente, las acciones formativas en turismo siempre han sido genéricas y abstractas - una realidad que, además de desanimar al estudiante, también se traduce, al final del proceso formativo, en el desarrollo de prácticas turísticas significativamente homogeneizadas, con experiencias inmemorables.

Uno de los principales retos, en las bases territoriales, para la implementación de los objetivos y premisas de PNCT es la falta de Órganos de Gobierno local que permitan a los más diversos actores - incluyendo a los poderes públicos, las empresas, las comunidades tradicionales, los egresados de los cursos de turismo y las organizaciones de la sociedad civil - dialogar y establecer estrategias para la calificación local en turismo (SILVA, 2020).

Por ello, es indispensable crear, en las bases territoriales, redes de actores implicados en los procesos de cualificación en turismo. Estas redes son acuerdos - institucionalizados o no - que permiten a los actores directa o indirectamente involucrados con el turismo dialogar y, así, definir las mejores estrategias de calificación para la localidad, configurando el turismo que desean realizar, para sí mismos y para los visitantes, en el territorio. En este sentido, Silva y Fratucci (2019) señalan que las redes de base territorial deben ser inclusivas y democráticas, con el alcance de permitir que las acciones de calificación turística lleguen a todas las personas interesadas, especialmente a las más invisibles, como las comunidades tradicionales que, a pesar de tener interés y múltiples potencialidades para integrarse en los sistemas productivos del turismo, a menudo son olvidados al aplicar estrategias de calificación en turismo.

Por lo tanto, teniendo en cuenta las preguntas anteriores; y tomando como antecedente el propósito de este artículo, es fundamental entender la configuración de la Ruta Bioceánica para, a partir de entonces, pensar en una propuesta que permita la calificación en turismo en la localidad en cuestión, a la luz de las premisas de PNCT.

\section{LA RUTA BIOCEÁNICA Y EL POTENCIAL PARA EL TURISMO}

Considerada un instrumento prometedor de integración latinoamericana, la Ruta Bioceánica surgió en 2015, cuando los jefes de Estado de Brasil, Paraguay, Argentina y Chile firmaron la Declaración de Asunción, que tenía compromisos y expectativas multilaterales sobre la Ruta, cuyos puntos extremos serían Campo Grande (en el Estado de Mato Grosso do Sul, Brasil) y los Puertos de Antofagasta, Mejillones e Iquique, Chile (BRASIL, 2015).

Cabe destacar que el surgimiento de la Ruta Bioceánica se produjo con el objetivo primordial de crear un trazado vial que facilitará - en términos logísticos y aduaneros - el flujo de bienes y servicios entre los países sudamericanos, aumentando la competitividad del comercio internacional en la región (SILVA; FERES, 2021).

Al mismo tiempo, después de más de cinco años de institucionalización de la Ruta Bioceánica, se observa que uno de los mayores obstáculos para la materialización efectiva del proyecto es la construcción de un puente sobre el río Paraguay, que conectará la ciudad brasileña de Porto Murtinho (en el Estado de Mato Grosso do Sul, Brasil) con la ciudad paraguaya de Carmelo Peralta. Aunque los gobiernos de los dos países ya han establecido acuerdos para la construcción conjunta del puente, el proyecto se detuvo a principios de 2020 debido a la aparición global de la pandemia de Covid-19, en una dinámica en la que el cruce entre las dos ciudades se realiza en ferry (MATO GROSSO DO SUL, 2021). 
Aunque la materialización efectiva y "real" de la Ruta Bioceánica no se ha materializado, es importante destacar que el proyecto está rodeado de muchas expectativas, especialmente en lo que respecta a la generación de alternativas de desarrollo e integración multilateral para los países de América del Sur (ASATO; GONÇALVES; WILKE, 2019).

El turismo, aunque no esté incluido en los objetivos y metas expresados en la Declaración de Asunción, sigue siendo considerado una actividad estratégica para que la Ruta sea una forma de generar oportunidades de empleo, ingresos y desarrollo (CASTRO, 2019; SILVA; FERES, 2021).

Entre las modalidades turísticas que se pueden acometer en el contexto de la Ruta Bioceánica, cabe destacar (ASATO et al., 2019; MAMEDE et al., 2019; FERREIRA; CASTILHO; OLIVEIRA, 2019): (a) turismo de negocios y eventos, basado en el movimiento de personas interesadas en transacciones comerciales entre países sudamericanos; (b) el turismo histórico, que puede aprovechar el patrimonio histórico existente en la región, destinado - por ejemplo a la Guerra del Paraguay (1864-1870), que fue un acontecimiento importante en la historia de América del Sur; (c) el turismo cultural y étnico, que impregna la identidad e incluso los aspectos gastronómicos de los pueblos y comunidades sudamericanos, tan diversos desde el punto de vista cultural; (d) el turismo de aventura y grandes expediciones, con actividades de senderismo, escalada, ciclismo, rally y similares; (e) el turismo pesquero, incluida la pesca deportiva, que ya es una actividad muy prometedora en todo el curso del río Paraguay; ( $f$ ) el ecoturismo, basado en paisajes naturales muy singulares, como el Pantanal y el Cerrado brasileños, el Chaco paraguayo, incluso los Andes argentinos y chilenos; (g) el turismo de observación de la fauna y flora silvestres y, más concretamente, el turismo de observación de aves, (birdwatching), que es muy prometedor en función de las especies animales endémicas de América del Sur.

Al considerar el territorio brasileño de la Ruta Bioceánica, se percibe que Mato Grosso do Sul es uno de los estados brasileños más involucrados con el proyecto en cuestión, ya que alberga las ciudades de Campo Grande (punto de partida de la Ruta) y Porto Murtinho, este último ya en la frontera entre Brasil y Paraguay, considerado un punto nodal estratégico de integración bioceánica. Vale la pena señalar que el eje Campo Grande-Porto Murtinho tiene un importante potencial turístico, especialmente vinculado a los activos socioambientales del Pantanal brasileño. De todos modos, Silva y Fratucci (2019) señalan que la Ruta Bioceánica puede generar un conjunto promisor de externalidades positivas, impactando en el desarrollo turístico de localidades que, aunque no "atravesadas" por la Ruta, aún pueden recibir una mayor demanda de los productos turísticos del territorio. También según los autores, esta es la configuración de Bonito, un importante destino de ecoturismo en Mato Grosso do Sul y Brasil, que aunque no "pertenece" a la Ruta, todavía está previsto un mayor flujo de turistas al destino desde el momento en que el proyecto de integración bioceánica se extienda de manera efectiva.

Almeida, Teixeira y Figueira (2019), además de Lunas, Eichenberg y Le Bourlegat (2019) señalan que a pesar de los beneficios positivos de la Ruta Bioceánica, también es esencial considerar los impactos negativos de esta empresa, impactos que están vinculados principalmente a la posibilidad de degradación ambiental, caracterización errónea del territorio, las culturas y las identidades locales, por no mencionar las actividades no deseadas (como las prácticas de contrabando, tráfico, violencia y delitos menores) que pueden ocurrir si el proyecto de integración bioceánica se materializa sin más planificación.

En este particular, es importante destacar que la creación de una propuesta de calificación en turismo es una condición fundamental para la planificación del turismo en la Ruta Bioceánica. 
En cierto modo, las prácticas formativas en turismo en la Ruta deben, en términos generales (CASTRO, 2019): (a) brindar oportunidades para que los actores del territorio sean protagonistas de las prácticas turísticas; (b) permitir una mayor integración y colaboración transnacional, incluso al considerar el universo de la innovación y la tecnología, y; (c) preparar los destinos turísticos y los productos presentes a lo largo de la Ruta Bioceánica, con el fin de permitir la materialización de prácticas turísticas sostenibles y responsables.

Es importante destacar que la Ruta Bioceánica ya cuenta con una red de instituciones universitarias denominada UniRila - Red Universitaria de la Ruta de la Integración Latinoamericana (UEMS, 2021). Dentre esas instituciones incluyen la Universidad Católica Don Bosco (UCDB), la Universidad Estatal de Mato Grosso do Sul (UEMS), la Universidad Federal de Mato Grosso do Sul, el Instituto Federal de Mato Grosso do Sul (IFMS) y la Universidad para el Desarrollo del Estado y la Región del Pantanal (UNIDERP) - todas ellas en Brasil - así como instituciones universitarias en Paraguay, Argentina y Chile. En un comunicado, esta instancia de gobernanza ya elaborada e institucionalizada puede ser fundamental para la difusión de acciones formativas en el ámbito de la Ruta Bioceánica.

Sin embargo, ¿qué otras organizaciones también pueden participar en la red de calificación turística en la Ruta Bioceánica? ¿Qué temas y acciones son más urgentes, en términos de calificación, en el territorio brasileño de la Ruta Bioceánica, considerando que la calificación en turismo es algo estratégico y fundamental, según lo dispuesto por el PNCT? Finalmente, después de este trabajo hay un esfuerzo para comprender estos temas, a partir de la indicación de una propuesta de calificación en turismo para el territorio brasileño de la Ruta Bioceánica.

\section{PROCEDIMIENTOS METODOLÓGICOS}

La investigación empírica que permitió la recolección de datos para este artículo se debió a un proyecto desarrollado por un equipo de investigadores de la Universidad Federal de Mato Grosso do Sul (UFMS). En definitiva, el proyecto en cuestión pretendía recaudar subvenciones para la elaboración de estrategias que resultaran en la implementación, en las bases territoriales, de la Política Nacional de Calificación Turística (PNCT).

La investigación empírica tuvo básicamente tres fases secuenciales, que se produjeron entre julio/2020 y abril/2021, cuyas características fundamentales se expresan en el Cuadro 1.

Cuadro 1 - Fases de la investigación empírica

\begin{tabular}{|c|l|}
\hline 19. Fase & \multicolumn{1}{c|}{ Características } \\
\hline 20. Fase & $\begin{array}{l}\text { Se realizó una encuesta a los actores involucrados en el turismo en la Ruta Bioceánica. Se } \\
\text { estableció contacto con estos actores (por teléfono y/o correo electrónico), y se les dio la } \\
\text { oportunidad de participar en el proceso de investigación que resultaría en la elaboración } \\
\text { de una propuesta de calificación para la Ruta }\end{array}$ \\
\hline 3o. Fase & $\begin{array}{l}\text { Se llevaron a cabo ruedas de conversación (cuyos detalles serán expuestos más adelante), } \\
\text { con la perspectiva de elevar, de manera dialógica, la configuración de la oferta y la } \\
\text { demanda de calificación en turismo en el territorio brasileño de la Ruta Bioceánica }\end{array}$ \\
\hline $\begin{array}{l}\text { Después de la sistematización y análisis de las informaciones, se elaboró una propuesta de } \\
\text { calificación para el territorio brasileño de la Ruta Bioceánica, de manera que el producto } \\
\text { en cuestión fue validado con los propios actores que participaron en la investigación } \\
\text { empírica }\end{array}$ \\
\hline
\end{tabular}

Fuente: Elaborado por los autores

INTERAÇÕES, Campo Grande, MS, v. 22, n. 4, p. 1317-1334, out./dez. 2021. 
Como se menciona en el Cuadro 1, la estrategia, después del mapeo y los contactos con los actores relacionados con el turismo en la Ruta Bioceánica, fue llevar a cabo ruedas de conversación que, en resumen, se refieren a espacios en los que las personas establecen diálogos e intercambios entre sí, con el propósito de mostrar detalles del funcionamiento y desarrollo de un determinado fenómeno de la vida social (MÉLLO et al., 2007). En este sentido, Melo y Cruz consideran que:

Las ruedas de conversación son 'discursos' sobre ciertos temas discutidos por los participantes sin la preocupación por el establecimiento de un consenso, y las opiniones pueden convergir o divergir, provocando debate y controversia. Corresponde al mediador garantizar la participación igualitaria de todos, así como cumplir los criterios para estructurar el debate. (MELO; CRUZ, 2014, p. 33).

La rueda de conversación se considera un espacio de intercambio libre, colectivo y heterogéneo al agregar diferentes temas con historias singulares. Por esta razón, se convierte en una herramienta metodológica de investigación relevante para la descubierta en el área de las Ciencias Humanas y Sociales al intentar "[...] comprender la aparición y consolidación de eventos considerados “hechos' y/o 'problemas sociales'” (MELLO et al., 2007, p. 30).

Antes del inicio de las ruedas de conversación que se llevaron a cabo en el ámbito de la investigación, los participantes fueron informados sobre las características generales de la investigación. Además, hubo una preocupación por solicitar el permiso de todos para hacer la grabación de la rueda de conversación. Una vez que los participantes estuvieron plenamente de acuerdo, los investigadores proporcionaron la grabación, en audio y video, de los diálogos.

Así, las ruedas de conversación se realizaron de forma remota a través de la plataforma de videoconferencia Zoom. Esta opción se debió al contexto de la pandemia de Covid-19, que hizo imposible que los investigadores se reunieran de manera segura con los actores invitados a participar en la investigación. En términos operativos, fue un reto importante poder hacer compatible la agenda a todos los participantes de las ruedas, para que estuvieran conectados, en el mismo día y hora, sincrónicamente, para realizar la rueda de conversación.

Es importante destacar que las ruedas de conversación fueron conducidas a partir de un guión que contenía preguntas abiertas y flexibles que se relacionaban con: (a) la percepción del grupo sobre el turismo en el segmento brasileño de la Ruta Bioceánica; (b) las características de la oferta de calificación turística en el territorio de la Ruta Bioceánica; (c) las principales demandas de aumento de la actividad turística en el territorio objeto de análisis y; (d) los desafíos para las comunidades tradicionales que deben insertarse en la dinámica de calificación en el turismo en la Ruta. En posesión de las grabaciones de las ruedas de conversación, se proporcionó la transcripción completa de los diálogos y reflexiones que "surgieron" a lo largo de las discusiones de las ruedas.

Se realizaron un total de 6 (seis) ruedas de conversación con representantes de las siguientes instituciones, que están directa o indirectamente vinculadas a los procesos de calificación en turismo en el territorio brasileño de la Ruta Bioceánica: instancias de gobierno regional y local; Sistema S (incluyendo SEBRAE, SENAR y SENAI); asociaciones empresariales y de difusión del turismo local/regional; instituciones de enseñanza y de investigación que conforman UniRila; asociaciones de trabajadores del turismo y; Organizaciones de la Sociedad Civil (OSC) que trabajan con comunidades tradicionales que forman parte y/o tienen el potencial de actuar en el campo del turismo en la Ruta Bioceánica. 
Como camino metodológico de la investigación, adoptamos la materialidad discursiva - que llamamos discursividades - como instrumento de análisis, partiendo de un enfoque cualitativo y apoyándose en los estudios del Círculo de Bakhtin (2003), que propone el análisis dialógico del lenguaje como camino de interpretación y comprensión de los discursos.

Este enfoque consiste en la comprensión del lenguaje como una cuestión concreta del objeto de estudio en cuestión y su naturaleza es la interacción. El lenguaje es la mediación entre un "Yo" y el "Otro". Revela, refleja y retracta el mundo y las diversas formas de intervenir y construir en él. El lenguaje es un producto de la actividad humana colectiva; producto de las relaciones sociales, culturales e históricas, constituyendo no sólo conocimiento y valor, sino también de ética, estética, crítica, responsabilidad, respuesta en cada interacción social. "Sin el lenguaje humano del hombre no está constituido. La única forma apropiada de expresión de la vida auténtica del hombre es el diálogo inconcluso que tiene lugar en la enunciación" (BAKHTIN, 2003, p. 348).

Por lo tanto, las enunciaciones y sus relaciones dialógicas fueron elegidas como materia privilegiada para la búsqueda del proceso de comprensión e interpretación de los significados atribuidos por los autores de los discursos analizados, en los que lo que predomina es la perspectiva de la situación concreta, por una realidad social e histórica, considerando la enunciación dentro de su propio contexto. Los análisis sugeridos para pensar en el discurso es el de una práctica eminentemente social y de diálogo, intercambio, construcción, y no simplemente en función de "homogeneizar" ideologías, sino de ser capaz de producir singularidades (BAKHTIN, 2003).

Tras el análisis de los datos, a la luz de las discursividades, se elaboró la red de actores vinculados a los procesos formativos en turismo, al tiempo que se sistematizó la propuesta de calificación en turismo en la Ruta Bioceánica. Cabe destacar que ambos "productos" (red de actores y la propuesta de calificación) fueron presentados a todos los participantes de la investigación que, en una reunión también celebrada de forma remota, a través de la plataforma Google Meet, en abril/2021, validaron los productos en pantalla, presentando sugerencias, que se incorporaron a la investigación y dieron como resultado la indicación de referencias para calificación en turismo en la Ruta Bioceánica, según detalles que siguen este artículo.

\section{LA RED DE ACTORES Y LA PROPUESTA DE CALIFICACIÓN EN TURISMO EN LA RUTA BIOCEÁNICA}

La red de actores vinculados al turismo en la Ruta Bioceánica, como se muestra en la Figura 1 , presenta un conjunto de sinergias, intercambios e interrelaciones - institucionalizadas o no - que pueden fomentar, de forma muy prometedora, los procesos de cualificación en turismo. 
Figura 1 - Red de actores vinculados a la propuesta de calificación en turismo en la Ruta Bioceánica

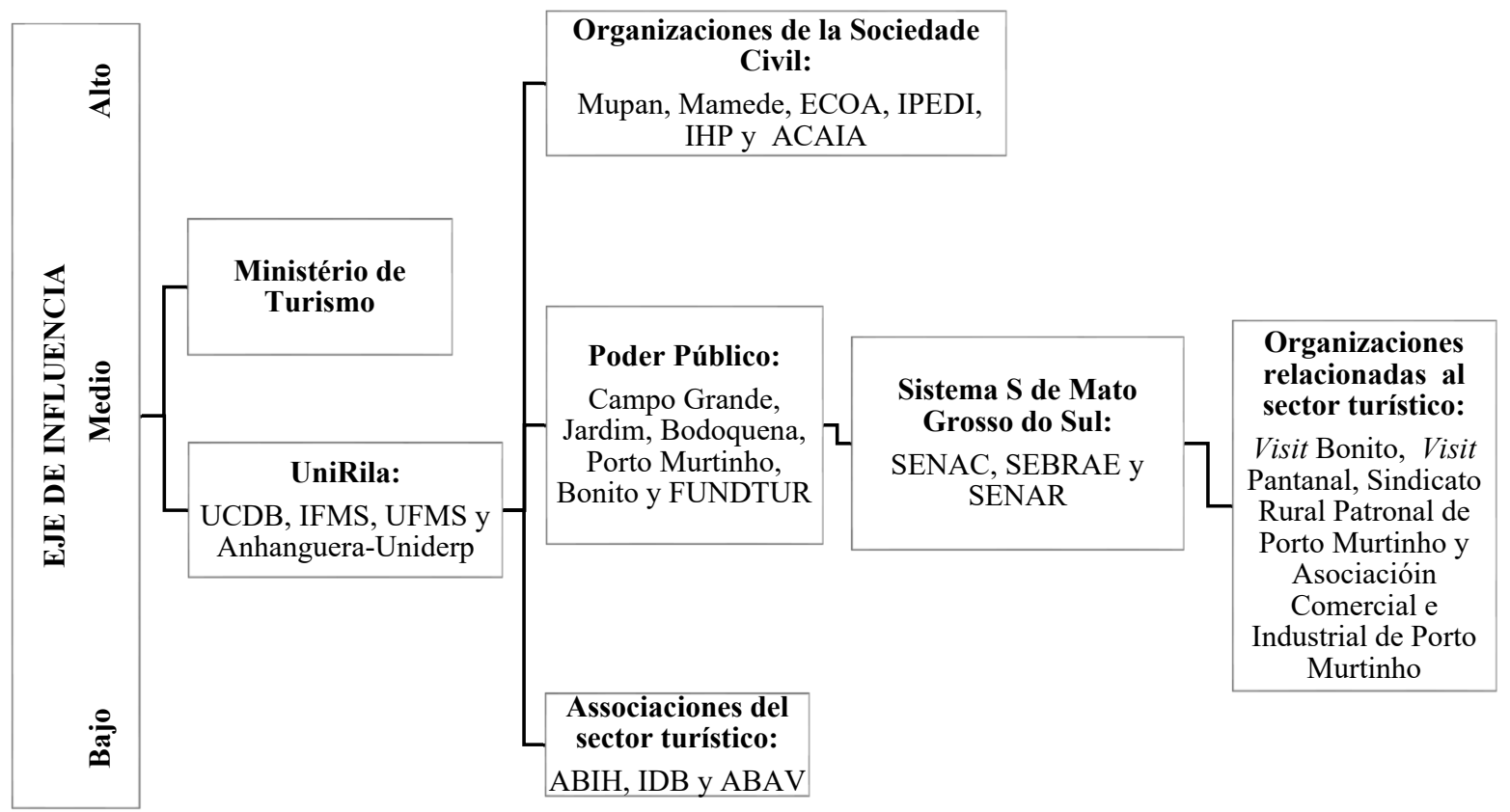

Fuente: Preparado por los autores.

Aunque todos los actores enumerados en la Figura 1 son importantes para la calificación en turismo, se pudo percibir que la influencia ejercida por cada uno de ellos es diferente, con el horizonte de la posible materialización, en un futuro próximo, de la propuesta de calificación en turismo para la Ruta Bioceánica. En otras palabras, esto significa que se percibió a lo largo de la investigación que ciertos actores son considerados estratégicos y, en este sentido, son fundamentales para que la propuesta de calificación se materialice, a la luz de las premisas de la Política Nacional de Calificación Turística (PNCT).

Fue curioso darse cuenta, a lo largo de la investigación, de que las organizaciones de la sociedad civil (OSC) demostraron ser fundamentales para la posible implementación de la propuesta de calificación en turismo en la Ruta Bioceánica. En una variedad de formas, estas organizaciones tienen una capacidad significativa en la red, mientras que tienen contactos muy fructíferos con las comunidades tradicionales que, con base en las premisas del PNCT, deben ser incluidas en el ámbito de los procesos de calificación en turismo para asegurar la diversidad de representatividad, también en los órganos de gobierno. Además, también es importante mencionar que las OSC's tienen un conjunto de proyectos y acciones en curso que, idealmente, pueden ser "utilizados" y/o re-significados como instrumentos para la difusión de acciones de calificación en el territorio brasileño de la Ruta Bioceánica.

Entre las OSC's que participaron en la investigación y son consideradas cruciales para la difusión de las acciones de calificación en el territorio brasileño de la Ruta Bioceánica, es posible mencionar: la Organización No Gubernamental (ONG) Ecología y Acción (ECOA); el de Instituto de Investigación de la Diversidad Intercultural (IPEDI); el Instituto Mamede- Investigación Ambiental y Ecoturismo; la Organización de la Sociedad Civil de Interés Público (OSCIP) Mujeres en Acción en el Pantanal (MUPAN) y Wetlands International; el Instituto Acaia Pantanal; además del Instituto del hombre Pantanero (IHP). 
Otro aspecto importante es la relevancia de las instituciones de enseñanza que conforman UniRila (Red Universitaria de la Ruta de la Integración Latinoamericana). Estas instituciones tienen una influencia significativa en la red principalmente porque: (a) reconocen el potencial turístico inherente a la Ruta Bioceánica; (b) emprenden proyectos de investigación y extensión con el tema de las múltiples cuestiones (incluido el turismo) que impregnan la Ruta; (c) reúnen al capital humano con formación técnica y conocimientos para la difusión de acciones de cualificación y; (d) disponen de infraestructura física que pueda utilizarse en el ámbito de la oferta de múltiples actividades formativas en el sector de turismo.

Sin embargo, se verificaron que las instancias del gobierno local y regional (municipios y gobierno del Estado de Mato Grosso do Sul), además de las instituciones del "Sistema S", aunque sean relevantes para la difusión de las acciones de calificación en la Ruta Bioceánica, no fueron plenamente sensibilizadas para que sean más efectivas y precisas en las acciones de calificación que incrementen, a corto plazo, la formación técnica de trabajadores centrada en la realidad del turismo en el territorio brasileño de la Rota. Eso también se aplica a las organizaciones empresariales vinculadas al sector turístico. Como resultado, todas estas instancias tienen - al menos por ahora - un poder de influencia medio en la red de actores vinculados a la propuesta de calificación en turismo en la Ruta Bioceánica.

Por último, también vale la pena mencionar el hecho de que las asociaciones del sector turístico - como la Asociación Brasileña de la Industria Hotelera (ABIH), el Instituto de Desarrollo de Bonito (IDB) y la Asociación Brasileña de Agencias de Viajes (ABAV), que participaron en esa investigación -, aunque son fundamentales para congregar a los agentes que conforman el comercio turístico en los territorios, aun así tienen poca participación en los procesos de estimulación a las acciones de calificación, sobre todo cuando se considera el contexto de la Ruta Bioceánica. Así, ese grupo de instituciones, en el momento de la investigación, presentó bajo poder de influencia en la red de actores vinculados a la calificación en turismo en la Ruta Bioceánica.

A lo largo del proceso de investigación que dio lugar a este artículo, se identificaron un conjunto de obstáculos (llamados "muro de obstáculos"), presentados en la Figura 2, desde la perspectiva de los actores de la red, que impiden una difusión más efectiva de los procesos de calificación en turismo en la Ruta Bioceánica. 
Figura 2 - "Muro de obstáculos" para la calificación en turismo en la Ruta Bioceánica

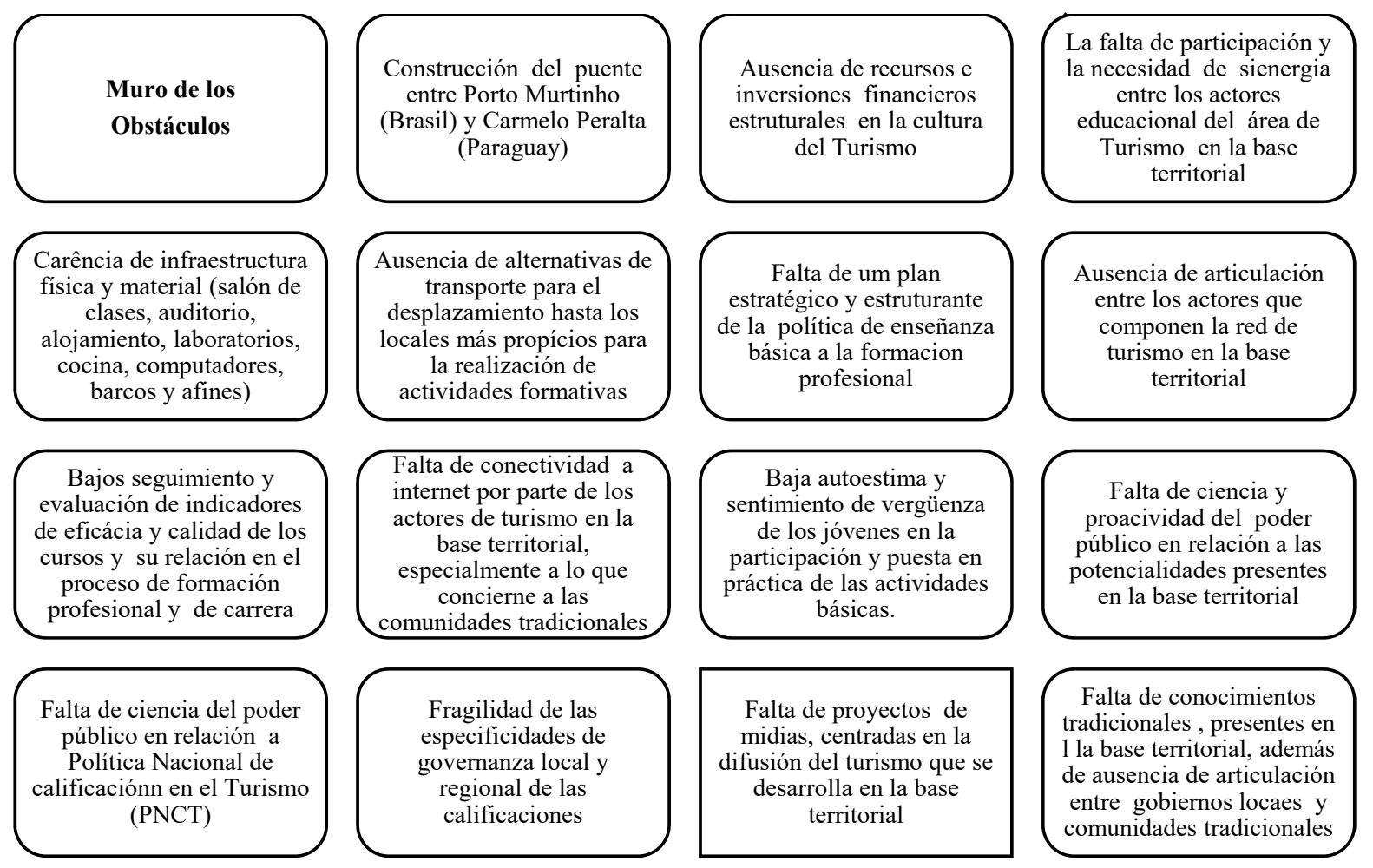

Fuente: Elaborado por los autores.

A partir del "muro de obstáculos" identificado y construido a partir de las discursividades presentadas por los actores que participaron en las ruedas de conversación, se elaboró una propuesta para la difusión de procesos formativos en turismo en la Ruta Bioceánica, teniendo como objetivo "transponer" el muro de obstáculos que impide la calificación en turismo en la región bajo análisis. Los "nudos" traídos en el muro presentaron convergencia a las brechas del PNCT, así como constituyeron un tejido social relacional fuerte e integrado que facilitó el diálogo entre las demandas de carácter político y técnico.

La propuesta de calificación en turismo en el territorio brasileño de la Ruta Bioceánica tiene como objetivo contribuir al desarrollo profesional y social de los trabajadores del turismo, a la mejora del proceso formativo en la prestación de servicios y productos turísticos, así como en el acceso y expansión de la empleabilidad a jóvenes, adultos, comunidades tradicionales y personas mayores de diferentes representaciones, a través de cursos y proyectos integrados, transversales, colaborativos e inclusivos.

Más específicamente, la propuesta de calificación en turismo en el territorio brasileño de la Ruta Bioceánica debe presentar los siguientes objetivos fundamentales (que, vale la pena decir, surgieron de las discursividades dadas a lo largo de las ruedas de la conversación): (a) fortalecer los niveles de escolaridad formal de los trabajadores del turismo; (b) servir a grupos históricamente marginados, como las comunidades tradicionales; (c) promover el empoderamiento de las mujeres y las personas de edad y; (d) estimular el espíritu empresarial.

Por ello, teniendo en cuenta los objetivos que surgieron de la propuesta de titulación en turismo en la Ruta Bioceánica, proponemos un conjunto de cursos y prácticas formativas en turismo sobre la Ruta Bioceánica, subdivididos en tres ejes estructurantes (Ecosistema Turístico, 
Gobernanza y Tecnología y Cultura), que se detallarán siguiendo este texto. Vale la pena señalar que los ejes en cuestión fueron sistematizados a partir del análisis de las discursividades de los actores a lo largo de las ruedas de conversación, a la luz del marco metodológico de Bakhtin (2003).

Con respecto al eje Ecosistema Turístico, vale la pena destacar la importancia de señalar este eje porque percibimos algunas demandas, que surgieron de las ruedas de conversación, sobre la ausencia del papel educativo y formativo de los cursos de turismo ofrecidos por las instituciones que operan en el territorio brasileño de la Ruta Bioceánica. En este sentido, cabe destacar que los cursos relacionados con el área de turismo presentan una desconexión de contenidos y metodología en relación con el contexto local y regional - un aspecto ya predicho por el propio PNCT.

Así se hicieron evidentes, en las discursividades de los actores turísticos de la Ruta Bioceánica, los aspectos relacionados con la alta deserción de los alumnos en los cursos, la fragilidad en las discusiones sobre hostelería, gestión y especificidades regionales en los planes de estudio de los cursos y en las titulaciones realizadas hasta el momento, por no hablar de la tímida articulación y diálogo entre la enseñanza básica y técnica y el mercado turístico, en la perspectiva de nuevos cambios de la sociedad y sus prácticas de consumo en el turismo.

Así, el lenguaje debe ser interdisciplinario y contextualizado como una actitud científica para analizar el turismo como un fenómeno social y dialógico, con el fin de construir proyectos y acciones de calificación más sólidas y consistentes, así como políticas públicas más inclusivas, coherentes y estables con las especificidades de la Ruta Bioceánica.

El objetivo fundamental de la calificación desde la perspectiva del eje Ecosistema Turístico es demostrar que el epicentro del turismo es de carácter humano, por lo que el proceso de comprensión de este fenómeno se llevará a cabo desde un lenguaje interdisciplinario y/o transdisciplinario en sus contenidos y metodologías para las demandas de calificación y formación, con el fin de hacer una lectura de planificación social de los actores y sus representaciones y la complejidad de las prácticas turísticas realizadas en el territorio y sus impactos a las comunidades.

Con respecto al eje Gobernanza y Tecnología, es importante destacar que este eje señalará las vías para el despertar de la administración pública como una incursión necesaria para las acciones turísticas en aparcería con las Organizaciones de la Sociedad Civil (OSC's). Dentro del Marco Normativo de las Organizaciones de la Sociedad Civil (MNOSC), mejor conocido como la Ley de Aparcerías, está regulado el Procedimiento de Manifestación de Interés Social (PMIS), que es un instrumento a través del cual las OSC's, en línea con los movimientos sociales y los ciudadanos, pueden presentar propuestas a las autoridades públicas para evaluar la posibilidad de realizar una Convocatoria Pública. Sin embargo, PMIS no garantiza la formalización de la aparcería de inmediato, pero habrá un movimiento local que presione el desempeño de la gobernanza local. Ambos deben ser conscientes de las posibles herramientas para una buena cooperación, y PMIS suele ser una última opción.

En cualquier caso, lo que se cambia urgentemente para que hayamos consolidado el turismo en el territorio brasileño de la Ruta Bioceánica, con respecto a las comunidades tradicionales y el conocimiento local, es la articulación con el gobierno local. Creemos que es deber del gobierno garantizar la equidad de oportunidades en el turismo. Mientras tanto, las comunidades locales necesitan: ser incluidas en los itinerarios turísticos; tener sus conocimientos y costumbres valoradas; además de contar con instrumentos (incluso en el área de las nuevas tecnologías y medios digitales) que permiten el surgimiento de acciones emprendedoras dentro del ámbito de 
las propias comunidades. Estos y otros pasos pueden consolidarse a través de una articulación entre los órganos de gobierno local y las OSC's, que tienen una historia de actuación con las comunidades, cada una con su propia singularidad y misión institucional.

Así, el objetivo principal del eje Gobernanza y Tecnología es: fortalecer la articulación entre las redes de OSC's que conforman el territorio brasileño de la Ruta Bioceánica con los órganos de gobernanza turística con el fin de establecer asociaciones de interés mutuo para la emancipación de los trabajadores del turismo que pertenecen a comunidades tradicionales.

Finalmente, en cuanto al eje Cultura, las discursividades de las ruedas de conversación mostraron la poca aproximación más allá de las brechas en las demandas de calificación que validan el conocimiento de las comunidades tradicionales y sus costumbres y relaciones de subsistencia. Esto también se ve reforzado en las discursividades de los actores cuando revelan la baja escolaridad de jóvenes y adultos, además del éxodo rural de mujeres trabajadoras en el turismo, al percibir la relación de desconocimiento del patrimonio cultural por parte de estas comunidades, el poder público y la iniciativa privada, así como la baja autoestima y sentimiento de vergüenza de los jóvenes en la implicación y puesta en marcha de las actividades básicas del territorio, tales como la pesca, la conducción del ganado, la recolección y procesamiento de frutas y la fabricación de productos artesanales.

El rescate de los conocimientos tradicionales sirve de apoyo para la implementación de sistemas de gestión, políticas públicas, agregación de ingresos y generación de conocimiento técnico-científico para la sociedad desde la perspectiva de la sociobiodiversidad, contribuyendo significativamente a la reducción de las desigualdades sociales y la conservación del medio ambiente. Los procesos de cooperación, comunicación intra e intergeneracional, autosuficiencia y contención de la explotación de los recursos y respeto a la naturaleza, confieren caminos de valorización a los conocimientos y prácticas populares, reconociendo a la diversidad cultural y simbólica existente en el territorio brasileño de la Ruta Bioceánica, recursos aún inconexos y poco articulados.

Por ello, para que las comunidades se inserten en el ámbito del turismo en la Ruta Bioceánica, creemos que el objetivo fundamental del eje temático Cultural es sensibilizar y encontrar caminos activos para (re) conocer la cultura y su relación con el turismo como política pública, con el fin de fortalecer la acción y el empoderamiento de todos los actores sociales involucrados en el proceso, dentro de una relación más equilibrada en el ámbito relacional de los poderes en las decisiones públicas.

Por lo tanto, tomando como perspectiva los aspectos presentados anteriormente, propusimos un conjunto de acciones de calificación, con el objetivo de cumplir con las metas fundamentales de cada uno de los ejes temáticos (Ecosistema Turístico, Gobernanza y Tecnología y Cultura) de esta propuesta de calificación en turismo para el territorio brasileño de la Ruta Bioceánica. 
Figura 3 - Acciones de calificación basadas en ejes temáticos

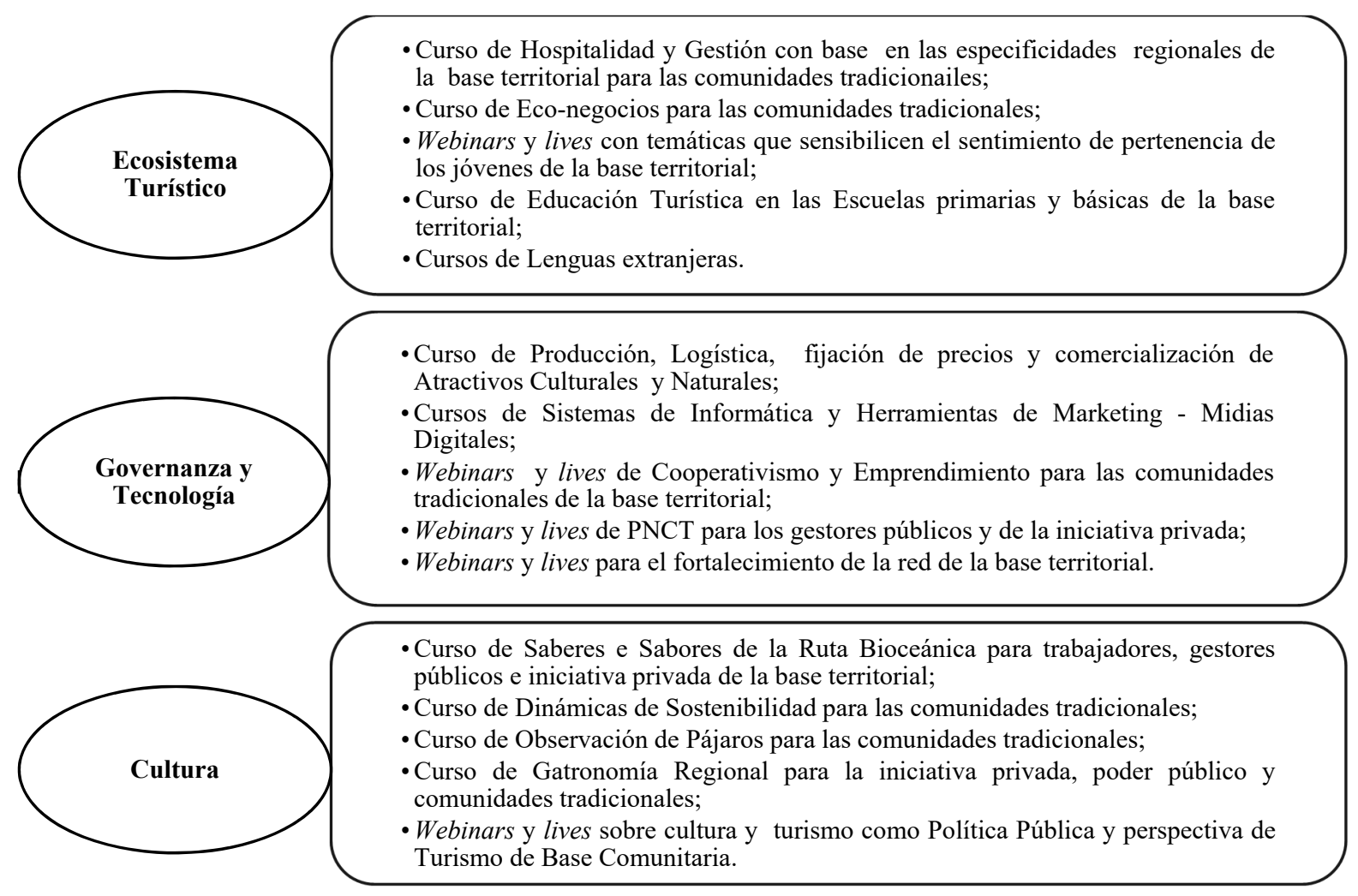

Fonte: Elaborado por los autores.

Es natural que las acciones de calificación puntuadas anteriormente sean desafiantes y complejas, requiriendo un conjunto de estrategias transversales que permitan, de manera más adecuada, la consecución de los objetivos de la propuesta de calificación en turismo. En cualquier caso, entendemos que las acciones bajo análisis son poderosas $y$, una vez implementadas, permitirían, de manera sinérgica y poderosa, la (re)significación de la condición de subalternidad y vulnerabilidad de muchos de los grupos y comunidades tradicionales que se pueden lograr con esta propuesta de calificación en turismo en el territorio brasileño de la Ruta Bioceánica.

\section{CONSIDERACIONES FINALES}

Este artículo, en términos generales, presentó una propuesta - con un conjunto de acciones de calificación - que puede catalizar los procesos formativos de los trabajadores del turismo en el territorio brasileño de la Ruta Bioceánica, contribuyendo potencialmente a la promoción, difusión y aumento de la competitividad de la actividad turística en la región bajo análisis.

Fue evidente, a lo largo del proceso de investigación que apoyó este texto, que las acciones de calificación en turismo en el territorio brasileño de la Ruta Bioceánica se subdividen en tres ejes temáticos: (a) Ecosistema Turístico, que sacó a la luz la importancia de los cursos turísticos teniendo en cuenta procesos metodológicos y pedagógicos más coherentes con las realidades locales, como ya ha señalado en la Política Nacional de Calificación en Turismo (PNCT); (b) Gobernanza y Tecnología, cuyo lema era la pertinencia de los órganos de gobernanza local, con la participación de las Organizaciones de la Sociedad Civil (OSC's) para que los procesos de capacitación sean más amplios y; (c) Cultura, cuyo alcance tiene por objeto arrojar luz sobre la importancia de 
los medios de vida, los hábitos y las identidades locales, especialmente manifestados por las comunidades tradicionales, que también deberían beneficiarse de las externalidades positivas del turismo en el territorio brasileño de la Ruta Bioceánica.

Quedó claro, a lo largo de las reflexiones de este texto, que los órganos de gobierno local son cruciales para la potencial materialización de las acciones de calificación, a la luz de esta propuesta y de las premisas fundamentales del PNCT. Mientras tanto, entendemos que un mayor contacto entre los gobiernos locales/regionales y los actores turísticos - incluyendo las OSC's - es esencial para que las políticas públicas, acciones y estrategias para la difusión del turismo se produzcan de una manera dialogada, sostenible y adherente con las características socioculturales locales.

Otro punto que es fundamental destacar es la relevancia de la Red Universitaria de la Ruta de integración latinoamericana (UniRila) como instancia de gobernanza ya institucionalizada en el ámbito de la Ruta y que, idealmente, puede representar un punto de apoyo fundamental de la red para que las acciones de calificación se difundan de manera más efectiva.

Por último, pero no menos importante, dejar constancia de la necesidad de que las comunidades tradicionales se inserten en el ámbito de las acciones de calificación en el territorio brasileño de la Ruta Bioceánica, a fin de permitir el turismo, también liderado por estas comunidades tradicionales, es marcadamente experiencial e inclusiva, elevó la Ruta a un nivel diferenciado dentro de los mercados turísticos (trans)nacionales.

\section{REFERENCIAS}

ALMEIDA, Luciane Pinho; TEIXEIRA, Léia Lacerda; FIGUEIRA, Kátia Cristina Nascimento. A importância do estudo dos impactos sociais junto às comunidades locais dos territórios que integram o Corredor Rodoviário Bioceânico. Interações, Campo Grande, MS, v. 20, n. especial, p. 285-96, 2019. http://dx.doi. org/10.20435/inter.v20iespecial.2590

ASATO, Thiago Andrade; CONSTANTINO, Michel; DORSA, Arlinda Cantero; MARIANI, Milton Augusto Pasquotto. Rota de Integração Latino-Americana (RILA) para o desenvolvimento turístico. Interações, Campo Grande, v. 20, n. especial, p. 45-56, 2019. https://doi.org/10.20435/inter.v20iespecial.1994

ASATO, Thiago Andrade; GONÇALVES, Débora Fittipaldi; WILKE, Erick Pusck. Perspectivas do Corredor Bioceânico para o desenvolvimento local no Estado de MS: o caso de Porto Murtinho. Interações, Campo Grande, MS, v. 20, n. especial, p. 141-57, 2019. http://dx.doi.org/10.20435/inter.v20iespecial.2476BAKHTIN, Mikhail M. Estética da criação verbal. São Paulo: Martins Fontes, 2003.

BRASIL. Ministério de Relações Exteriores. Declaração de Assunção sobre corredores bioceânicos. Brasília, DF: Ministério de Relações Exteriores, 2015.

BRASIL. Ministério do Turismo. Política Nacional de Qualificação no Turismo (PNQT). Brasília, DF: MTUR, 2018.

BRASIL. Plano Nacional de Turismo 2018-2022. Brasília, DF: Governo Federal, 2018.

CASTRO, João Carlos, P. Turismo como instrumento dinamizador do Corredor Rodoviário Bioceânico. Interações, Campo Grande, MS, v. 20, n. especial, p. 19-29, 2019. http://dx.doi.org/10.20435/inter. v20iespecial.2419

CATRAMBY, Teresa Cristina Viveiros; COSTA, Stella Regina Reis. Qualificação profissional em turismo como fator de competitividade do setor. Caderno Virtual de Turismo, Ilha do Fundão, v. 4, n. 3, p. 26-34, 2004. 
FERREIRA, Magali Luzio; CASTILHO, Maria Augusta; OLIVEIRA, Edilene Maria. Brasil, Paraguai, Argentina e Chile / Rota Bioceânica: relações culturais no território vivido. Interações, Campo Grande, MS, v. 20, n. especial, p. 69-89, 2019. http://dx.doi.org/10.20435/inter.v20iespecial.2299

LUNAS, Maria Cristiane; EICHENBERG, Fábio; LE BOURLEGAT, Cleonice. Desafios do planejamento turístico do Corredor Bioceânico: indicadores de desenvolvimento sustentável do turismo. Desenvolvimento, Fronteiras e Cidadania, Ponta Porã, MS, v. 3, n. 2, p. 154-72, 2019.

MAMEDE, Simone; BENITES, Maristela; ESQUIVEL, Alberto; CLAY, Robert; MERIGHI, Geancarlo L.; ALHO, Cleber José R. Turismo de observação de aves no Chaco: oportunidades e desafios ao Corredor Bioceânico, segmento Brasil/Paraguai. Interações, Campo Grande, MS, v. 20, n. especial, p. 159-77, 2019. http://dx.doi. org/10.20435/inter.v20iespecial.2386

MATO GROSSO DO SUL (Estado). Após suspensão de licitação, governo requisita novo cronograma para obra de ponte sobre Rio Paraguai. Portal do Governo de Mato Grosso do Sul, Campo Grande, 2021. Disponible em: http://www.ms.gov.br/apos-suspensao-de-licitacao-governo-requisita-novo-cronogramapara-obra-de-ponte-sobre-rio-paraguai/. Acesso em: 30 abr. 2021.

MÉLLO, Ricardo Pimentel; SILVA, Alyne Alvarez; LIMA Maria Lucia Chaves; DI PAOLO, Ângela F. Construcionismo, práticas discursivas e possibilidades de pesquisa em psicologia social. Psicologia \& Sociedade, Belo Horizonte, v. 19, n. 3, p. 26-32, 2007. https://doi.org/10.1590/S0102-71822007000300005

MELO, Marcia Cristina Henares; CRUZ, Gilmar de Carvalho. Roda de conversa: uma proposta metodológica para a construção de um espaço de diálogo no ensino médio. Imagens da Educação, v. 4, n. 2, p. 31-39, 2014. https://doi.org/10.4025/imagenseduc.v4i2.22222

SILVA, Ana Karolina Morais; FERES, Carla Paulino da Costa. Integração de infraestrutura na América do Sul: o papel geopolítico do projeto do Corredor Rodoviário Bioceânico. Revista de Geopolítica, Natal, v. 12, n. 1, p. 33-47, 2021.

SILVA, Ivan Conceição Martins. Políticas públicas e qualificação profissional em turismo: uma análise das metodologias de pesquisa. Revista Acadêmica Observatório de Inovação do Turismo, Rio de Janeiro v. 14, n. 3, p. 94-117, 2020. https://doi.org/10.17648/raoit.v14n3.6027

SILVA, Ivan Conceição Martins. A dimensão política da qualificação profissional na política pública nacional de qualificação profissional em turismo. 2019. 262f. Dissertação (Mestrado em Turismo) - Faculdade de Turismo e Hotelaria, Universidade Federal Fluminense (UFF), Niterói, RJ, 2019.

SILVA, Ivan Conceição Martins; FRATUCCI, Aguinaldo Cesar. A dimensão espacial das políticas públicas de qualificação profissional em turismo. In: TURISMO em foco. Belo Horizonte: Poisson, 2019, p. 62-72. V. 2

UNIVERSIDADE ESTADUAL DE MATO GROSSO DO SUL. UniRila - integrar caminhos, povos e conhecimentos no coração da América Latina. [s.I.]: UEMS, 2021. Disponible em: http://www.uems.br/midiaciencia/ unirila/. Acceso el: 30 abr. 2021.

WORLD TRAVEL \& TOURISM COUNCIL. Brazil - 2020 annual research: key highlights. London, United Kingdom: WTTC, 2021.

\section{Sobre los autores y las autoras:}

Dyego de Oliveira Arruda: Postdoctorado en Administración por la Universidad Federal de Mato Grosso do Sul (UFMS). Doctorado en Administración Organizacional por la Universidad de São 
Paulo (USP). Máster en Administración y Grado en Ciencias Económicas por la UFMS. Profesor titular en el Centro Federal de Educación Tecnológica Celso Suckow da Fonseca, Rio de Janeiro (CEFET/RJ), campus de Valença, docente en los cursos de pregrado en Administración e Ingeniería de Alimentos, y profesor titular en el Programa de Posgrado (nivel de máster académico) en Relaciones Étnico-Raciales (PPRER) del CEFET/RJ (campus Maracanã), trabajando en la línea de investigación "Pensamiento y Políticas Públicas: Dimensiones Institucionales de las Relaciones Étnico-Raciales”. E-mail: dyego.arruda@gmail.com, Orcid: https://orcid.org/0000-0002-9514-284X

Patricia Zaczuk Bassinello: Doctorado en Ciencia, Tecnología y Sociedad por la Universidad Federal de São Carlos (UFSCar). Profesora del plantel permanente del campus Aquidauana de la Universidad Federal de Mato Grosso do Sul (UFMS), impartiendo docencia en la carrera de bachillerato en Turismo y en el Programa de Posgrado en Estudios Culturales. E-mail: patrícia.zaczuk@ufms.br, Orcid: http://orcid.org/0000-0003-4983-9221

Carla Cristina de Souza: Estudiante de Doctorado en Psicología en la Universidad Católica Dom Bosco (UCDB), en la línea de investigación Políticas Públicas, Cultura y Producción Social. Máster en Antropología Social y grado en Ciencias Sociales por la Universidad Federal de Mato Grosso do Sul (UFMS). Miembro de los grupos de investigación: Psicología de la Salud, Políticas de Cognición y Subjetividad (UCDB); e Improprias - Grupo de Investigación en Género, Sexualidad y Diferencias (UFMS). E-mail: carlinhacdsouza@gmail.com, Orcid: https://orcid.org/0000-0002-6948-642X

Milton Augusto Pasquotto Mariani: Postdoctorado en Administración por la Universidad de São Paulo (USP). Doctorado en Geografía Humana por la USP. Máster en Historia Social por la Pontificia Universidad Católica de São Paulo (PUC-SP). Grado en Geografía por la Universidad Estatal Paulista Júlio de Mesquita Filho (UNESP). Profesor titular de la Universidad Federal de Mato Grosso do Sul (UFMS), acreditado en los programas de posgrado: Máster en Administración, Ciencias Contables y Estudios Fronterizos. E-mail: miltmari@terra.com.br, Orcid: https://orcid.org/0000-0001-9485-0150 